\title{
EVALUACIÓN DE LOS INTERESES BÁSICOS ACADÉMICO PROFESIONALES DE LOS ESTUDIANTES DE SECUNDARIA
}

\section{EVALUATION OF THE BASIC INTERESTS ACADEMIC AND PROFESSIONAL IN THE SECONDARY STUDENTS}

\author{
Vicente Hernández Franco*
}

Universidad Pontificia de Comillas

\begin{abstract}
RESUMEN
Este trabajo se enmarca en el contexto del estudio de los intereses profesionales de los estudiantes de secundaria en dos momentos críticos para la elección de continuidad de estudios: cuarto de ESO y segundo de Bachillerato. Nos presenta la distribución del área vocacional de interés preferente de una muestra de 1250 estudiantes, entendida como aquella en la que el estudiante encuentra los estudios o profesión que le gustaría realizar en un futuro próximo. Analiza el efecto sobre los intereses profesionales de las variables de decisión vocacional, expectativas vocacionales de logro y etapa.
\end{abstract}

Palabras clave: intereses profesionales, evaluación de intereses, indecisión vocacional, expectativas vocacionales de logro.

\begin{abstract}
This work is framed in the context of the study of the professional interests of the secondary students in two critical moments for the election of continuity of studies: when concluding the obligatory secondary and when finishing the high school This work present the distribution of the vocational area of the preferable interest of the 1250 students' sample, defined as that in which the student finds the studies or profession that he would like to carry out in a next future. It also analyzes the effect on the professional interests of the variables of vocational decision, vocational achievement expectations and stage.
\end{abstract}

Key words: vocational interest; interest assessment; vocational indecision, vocational achievement expectations.

* Vicente Hernández Franco es Director del I.C.E. y Profesor en la Facultad de Ciencias Humanas y Sociales de la Universidad Potificia de Comillas. 


\section{Marco teórico}

Los intereses profesionales han sido el fundamento de la psicología vocacional y de la orientación profesional desde comienzos del siglo XX. Entre los pioneros en el estudio de los intereses profesionales se encuentran Fryer (1931), Kuder (1939), Strong (1943) y Darley y Hagenah (1955). Los intereses vocacionales han jugado un papel muy importante en el asesoramiento vocacional desde el surgimiento de la Orientación Profesional a comienzos del siglo XX. Parsons en 1909 indicó que la elección vocacional incluía el conocimiento que uno tiene sobre sí mismo, el conocimiento que uno tiene sobre las ocupaciones, así como las relaciones entre ambos. Adquirir el conocimiento de uno mismo supone el trabajo de conocer y entender las propias actitudes, capacidades, intereses y limitaciones que afectan a cada sujeto. Strong (1943) discutió la función que los intereses profesionales desempeñan en el proceso de asesoramiento vocacional. En la actualidad, los cuestionarios sobre intereses vocacionales se utilizan con gran asiduidad, y tanto las condiciones de aplicación como los resultados del uso de dichos cuestionarios constituyen uno de los tópicos más tratados por los orientadores profesionales.

La evaluación de los intereses profesionales se remonta a los años veinte en los que se inició un trabajo en el Instituto de Tecnología de Carnegie. Poco después, Strong (1927), publicó su primer inventario "Strong Vocational Interest" y Kuder (1939) publica su "Kuder Preference Record Personal, Form-A'. La evaluación de los intereses profesionales ha recibido una atención constante desde los trabajos pioneros de estos autores.

Es conocida la distinción que hacen Super y Crites (1962) acerca de los cuatro procedimientos tradicionales de obtener información sobre los intereses vocacionales: expresados, manifiestos, medidos a través de tests psicométricos e intereses inventariados. Los más utilizados por su probada fiabilidad y validez durante más de setenta años en el ámbito de la Psicología Vocacional son los cuestionarios de intereses inventariados junto con la exploración de los intereses expresados, también denominados preferencias vocacionales (Rocabert, 1987). De un lado los inventarios de intereses recogen un conjunto amplio de cuestiones que hacen referencia a actividades profesionales del tipo: "Reparar electrodomésticos" o "Poner inyecciones y vacunas". Estas cuestiones a su vez están organizadas en grupos vocacionales de clasificación como "Técnica Aplicada" o "Sanidad" sobre las que se le pide al individuo que manifieste en una escala su grado de rechazo o interés. Después se suman las respuestas obteniendo la puntuación directa del grado de interés del estudiante en cada uno de los grupos profesionales del cuestionario. Estas puntuaciones se bareman normalmente en percentiles y se obtiene un perfil de los intereses del estudiante cuando se le compara con un grupo de referencia. Estos grupos normativos pueden ser bien los estudiantes de su mismo curso o bien muestras empíricas de profesionales, por ejemplo de Enfermería, Ingenieros, Maestros, etc. La puntuación de cada sujeto se interpreta, pues, en relación con las diferencias interindividuales, es decir, las obtenidas entre sus resultados y los del grupo normativo que sirve de referencia para la comparación. De este modo el estudiante puede dimensionar a través de este "baremo externo" cual es su grado de interés hacia un grupo profesional al comparar sus puntuaciones con las que presenta una determinada comunidad profesional. Por tanto, los perfiles inventariados nos muestran las preferencias relativas del grado de interés del estudiante hacia los distintos grupos vocacionales entre sí.

Por otro lado los intereses expresados hacen referencia a cuestiones ocupacionales especificas del tipo: “¿En que profesión concreta te gustaría trabajar? o bien “¿Qué carrera te 
gustaría estudiar en la Universidad cuando acabes el Bachillerato? Hansen (1984) y Borgen (1986) realizaron sendas revisiones de la investigación pertinente sobre la comparación entre los intereses inventariados y expresados. El primero de estos investigadores concluye que existe una pequeña relación entre intereses inventariados e intereses expresados. Sin embargo, el segundo autor indicó que tanto los intereses inventariados como los expresados serían igualmente predictivos de la subsiguiente elección ocupacional. Athanasou y Cooksey (1993) revisaron los estudios sobre intereses expresados. Indicaron que un análisis de catorce estudios presentaba una media de la correlación igual 0.46 entre intereses expresados e intereses inventariados; a su vez encontraron que las diferencias intraindividuales eran claras en lo que a intereses expresados se refiere; y que las diferencias en las correlaciones estaban influenciadas por el uso de diferentes inventarios. Más allá de las simples comparaciones, Hansen(1984) sugirió la integración de intereses inventariados y expresados en los procesos de orientación profesional, y Borgen(1986) sugirió examinar más profundamente la interacción entre estos dos tipos de medida de los intereses. Por ejemplo, Laing, Swaney, y Prediger (1984) encontraron que los individuos preferían más una determinada actividad ocupacional si sus intereses inventariados eran congruentes con sus intereses expresados.

Cuanto mayor sea la congruencia entre los intereses autoestimados (expresados) y los medidos mediante inventarios (inventariados), tanto mayor es la madurez vocacional (Castaño, 1983: 229). Cuando los intereses expresados e inventariados son congruentes, lo cual es de esperar en la mayoría de las ocasiones (Apostal, 1985), la validez predictiva de la combinación de ambas medidas de los intereses es muy alta llegando a un 60\% (Bartling y Hood, 1981; Borgen y Seling, 1978). Sin embargo, cuando intereses expresados e intereses inventariados no son congruentes, los intereses expresados alcanzan de dos a cuatro veces más validez predictiva de cara a la elección vocacional cuando se comparan con los intereses inventariados (Bartling y Hood, 1981).

Podemos concluir que los intereses expresados contienen un componente motivacional, reflejan el proceso de una reflexión del individuo que precede a toda elección y de su jerarquía de prioridades, y consecuentemente sirven de guía de la conducta vocacional (Savickas, 1995). Darley y Hagenah (1955) ya pusieron de manifiesto que en la expresión directa de sus intereses profesionales, los individuos sintetizan sus percepciones sociolaborales sobre el acceso al empleo y reflejan de este modo sus aspiraciones personales. Existe una evidencia cada vez mayor en las investigaciones que comparan ambos procedimientos de medida, que concede una superior validez predictiva a los intereses expresados, frente a los intereses inventariados (Borgen y Seling, 1978; Dolliver, 1969).

Nuestras investigaciones anteriores (Hernández, 2001) han puesto de manifiesto la necesidad de medir conjuntamente tanto los intereses inventariados como los intereses expresados para estimar adecuadamente su congruencia como indicador de la fiabilidad intrasujeto de cara a su función en los procesos de orientación profesional. La aplicación simultanea de un inventario de intereses junto con un cuestionario de intereses expresados homologo abre la posibilidad en la práctica de obtener un indicador de la fiabilidad intrasujeto con los datos recogidos por ambos cuestionarios, a partir de la comparación de las similaridades entre ambos perfiles $k$, mediante las técnicas de análisis de distancias (Rivas, 1995: 102) disponibles en SPSS. Esto es, mediante la formula: $r_{a b}=1-\left(D_{a b}^{2} / 2 k\right)$, podemos expresar la relación de cero a uno entre los dos perfiles homólogos de intereses expresados e inventariados, que puede resultar de gran utilidad para los orientadores al disponer de una eficiente 
estimación empírica de la congruencia vocacional de los estudiantes en sus procesos de reflexión y toma decisiones vocacionales.

Gottfredson (1986) propone las siguientes consideraciones para la correcta utilización de los inventarios de intereses en el marco de un programa de orientación:

a) Los cuestionarios deben ser empleados en el marco de un tratamiento o proceso de orientación.

b) Los cuestionarios de intereses así como su material interpretativo constituyen auténticos programas de intervención, la especificidad de dichos programas difiere de un inventario a otro.

c) Los cuestionarios de intereses son más útiles cuando forman parte de un proceso de selección de carrera genérico que para reconocer las dificultades en la elección de una carrera concreta.

d) Los métodos de intervención basados en el diagnóstico de los intereses, deben estar en correspondencia con las metas especificas del programa de orientación establecido.

e) Las puntuaciones de los cuestionarios de intereses son útiles en el diagnóstico sobre si la carrera elegida es la más satisfactoria para el sujeto y si no es así buscar posibles explicaciones.

f) Los materiales interpretativos que acompañan a los cuestionarios de intereses, pueden ser aplicables en el tratamiento de algunos problemas de indecisión vocacional que aparecen en el proceso de elección de estudios académicos o profesionales.

g) Los cuestionarios de intereses son importantes en el desarrollo de nuevas alternativas cuando los sujetos deben tomar decisiones comprometidas acerca de su futuro académico y laboral.

En definitiva, podemos afirmar con Rocabert (Rivas, 1995: 206) que el análisis de los intereses vocacionales debe incluirse siempre al comienzo de un programa de intervención para la exploración de carrera, entendida ésta como el conjunto de actividades que se acomete para adquirir información que ayudará a la elección, preparación y entrada en una ocupación, y a ajustarse y progresar en la misma (Jordan, 1963). La exploración es considerada como una etapa precursora de la cristalización e implementación de la elección (Tiedeman, 1961). También es considerada como la actividad central que se requiere para poder tomar una decisión satisfactoria (Gelatt, 1962). Ésta es sin duda la tendencia dominante hacia la que se dirige la utilización de los cuestionarios de intereses y en la que se enmarcan nuestras aportaciones en este trabajo: no tratamos de evaluar los intereses de una forma meramente descriptiva y estática, sino también, intentamos que dicha evaluación sea un estímulo facilitador de la exploración de la conducta vocacional del asesorado, en una línea mas cualitativa, entendido como estímulo para la autoexploración y la explicitación y discusión de los valores vocacionales implícitos en las distintas alternativas de carrera.

\section{Objetivos}

Entendemos como "área vocacional de intereses básicos académico profesionales" o simplemente como "área vocacional" un conjunto de estudios y profesiones que comparten 
aspectos comunes entre sí, que les diferencian a su vez de las otras áreas y que son percibidas por los estudiantes holísticamente, como un todo con identidad y significatividad sociolaboral propia. Asimismo entendemos como "área vocacional de interés preferente" aquella donde se encuentran los estudios o profesión que el estudiante desea realizar en un futuro próximo. Como objetivo general de nuestro trabajo, buscamos explorar descriptivamente la distribución de las áreas vocacionales de interés preferente de los estudiantes de secundaria en dos momentos críticos de elección en nuestro sistema educativo: cuarto de secundaria y segundo de bachillerato. Finalmente, queremos explorar las diferencias en los intereses profesionales de los estudiantes de secundaria en función tanto de su grado de decisión vocacional como de sus expectativas vocacionales de logro y de la etapa educativa en la que se encuentran.

\section{Hipótesis}

Considerando el objetivo general que hemos indicado anteriormente y los objetivos específicos recién enunciados, en este estudio nos planteamos la comprobación de las siguientes hipótesis de trabajo:

Primera. La información facilitada por el CIBAP permite a los estudiantes de secundaria a partir de su perfil de intereses expresados, identificar su "área vocacional de interés preferente".

Segunda. Los intereses de los estudiantes tanto de cuarto de ESO como de segundo de Bachillerato se encuentran definidos entre una y tres áreas vocacionales y es muy bajo el porcentaje de estudiantes indefinidos vocacionalmente en estos cursos.

Tercera. No existen diferencias significativas en el grado de decisión vocacional de los estudiantes de cuarto de ESO con relación a los de segundo de Bachillerato.

Cuarta. Los estudiantes de secundaria presentan unas altas expectativas de logro hacia su área vocacional de interés preferente.

Quinta. Los estudiantes de secundaria más determinados en su área vocacional de interés preferente, están significativamente más seguros de llegar a desempeñar los estudios o profesión de su interés que los pertenecientes al grupo de estudiantes indecisos.

Sexta. Existen diferencias significativas en las valoraciones de las áreas vocacionales de intereses básicos de los estudiantes de secundaria que cursan cuarto de ESO en relación con los de segundo de Bachillerato.

\section{Metodología}

\section{Diseño}

Sobre la base de los objetivos que pretendemos estudiar se trata de un estudio ex-postfacto. Desde la perspectiva del tipo de datos que se obtienen, el diseño de nuestro estudio responde a una estrategia de tipo transversal o trans-seccional en la que empleamos dos cohortes con grupos de sujetos distintos (cohorte $4^{\circ}$ ESO y cohorte $2^{\circ}$ Bachillerato). 


\section{Variables e instrumentos}

La variables contempladas en nuestro estudio son:

- Área vocacional de interés preferente: definida como aquella de entre las diecinueve áreas recogidas en el CIBAP donde se encuentran los estudios o profesión que al estudiante le gustaría realizar en un futuro próximo.

- Grado de decisión: expresa la situación del estudiante en el momento de su elección de área vocacional de interés preferente, ante la obligación de optar por sólo una de entre las diecinueve áreas sobre las que elegir: 1) Entre las que le son indiferentes; 2) Entre las que le gustan bastante; 3) Entre sus tres o más preferidas; 4) Entre sus dos preferidas; 5) Es su única preferida.

- Expectativas Vocacionales de Logro: expresa la creencia del sujeto sobre sus probabilidades para alcanzar trabajar en la profesión de su interés. Las categorías consideradas son las siguientes: 1) Pienso que nunca llegare a desempeñarla; 2) Creo que tengo muy pocas posibilidades; 3) Pienso que es probable que pueda realizarla; y 4) Tengo una gran seguridad en conseguirlo.

- Etapa: puede tomar los valores de $4^{\circ}$ de ESO y $2^{\circ}$ de Bachillerato.

La recogida de datos se efectuó a través de la técnica del cuestionario. El principal instrumento elaborado y validado para esta investigación fue el "Cuestionario de Áreas de Intereses Básicos Académico Profesionales: CIBAP” (Hernández Franco, 2001).

\section{Cuestionario de Áreas de Intereses Básicos Académico Profesionales (CIBAP-2000)}

El CIBAP-2000 parte de la premisa, claramente fundamentada en el marco de la teoría de la Psicología Vocacional durante los últimos setenta y cinco años (Betsworth y Fouad, 1997) que postula cómo el conjunto de actividades y ocupaciones de constatada validez transcultural (Rivas, 1988: 98) que puede realizar una persona en nuestras sociedades occidentales industrializadas, recogidas en los diferentes "Inventarios de Intereses" elaborados hasta hoy, se pueden sintetizar y clasificar (generalmente mediante técnicas de análisis factorial exploratorio) en unos grupos bastante estables o dimensiones denominadas en el ámbito de la literatura vocacional anglosajona "Intereses Básicos" (Days y Rounds, 1997) en la línea de "Las Escalas de Intereses Profesionales Básicos" (BISs) del Strong Interest Inventory (Harmon, Hansen, Borgen y Hammer,1994).

El CIBAP-2000 lo componen diecinueve Áreas Vocacionales de Intereses Profesionales Básicos. Operacionalmente, cada Área de Intereses Básicos, agrupa un conjunto de estudios y profesiones que comparten aspectos comunes entre sí, que les diferencian a su vez de las otras áreas y que son percibidas por los estudiantes holísticamente, como un todo con identidad y significatividad sociolaboral propia. Podríamos considerarlo como un cuestionario de intereses expresados. A diferencia de otros cuestionarios de intereses expresados, donde se le pregunta a la persona directamente sobre cuestiones ocupacionales específicas del tipo: “¿En qué profesión concreta te gustaría trabajar?”, o bien “¿Qué carrera te gustaría estudiar en la Universidad cuando acabes el Bachillerato?”, o también "¿Qué ciclo formativo de formación profesional específica te gustaría realizar?”, este cuestionario pretende 
conocer el grado de preferencia e interés globalmente considerado hacia las que hemos denominado "Áreas Vocacionales de Intereses Profesionales Básicos" o sintéticamente áreas vocacionales.

En la selección de las diecinueve Áreas Vocacionales de Intereses Profesionales Básicos hemos partido fundamentalmente de los dieciocho grupos profesionales que componen el cuestionario de intereses inventariados "Cuestionario de Intereses Profesionales" (CIP-II) de García Mediavilla, L. et al. (1990), dada su alta fiabilidad y consistencia contrastada como instrumento de análisis de los intereses profesionales de los estudiantes de secundaria.

Una vez analizadas las definiciones operacionales que el CIP-II propone para cada uno de estos dieciocho grupos, decidimos añadir una nueva área no contemplada por este denominada "DEPORTE", considerando su relevancia y emergente caracterización vocacional (Pascual, 1997), recogida también en el "Inventario de Preferencias Profesionales" (IPP) de De la Cruz (1997) y en el "SAV-R" de Rivas, Rocabert y López (1998). En la redacción final del "CIBAP" hemos modificado el título de alguno de los grupos profesionales respecto del CIP-II para mejorar su comprensión, de acuerdo con las sugerencias recogidas en las entrevistas con los estudiantes que realizamos durante el estudio piloto, para facilitarles los informes personalizados de los resultados de intereses inventariados de este cuestionario.

En el CIBAP para cada Área se presenta una breve descripción explicativa de sus características más relevantes y a continuación una breve y ejemplificadora referencia de algunos de los estudios universitarios más representativos, así como la localización de las Familias Profesionales de Ciclos Formativos correspondientes a la nueva Formación Profesional Específica, para terminar reseñando algunas profesiones afines a dichos estudios y de distinto nivel de cualificación. En el cuadro 1 ofrecemos como ejemplo de esta estructura de contenidos el área vocacional de intereses básicos de SANIDAD.

\title{
CUADRO 1: Estructura de contenidos del área vocacional de intereses básicos de Sanidad.
}

\begin{abstract}
AREA VOCACIONAL DE SANIDAD:
Descripción: interés por profesiones relacionadas con la prevención, diagnóstico, curación y conservación de la salud tanto física como mental de las personas o los animales.
\end{abstract}

Estudios universitarios: Medicina en alguna de sus diferentes especialidades; Enfermería; Fisioterapia; Veterinaria; Farmacia; Psicología Clínica.

Ciclos Formativos: Familia Profesional de Sanidad.

Profesiones como: Médico de Familia; Pediatra; Cirujano; Dentista; Psiquiatra; Enfermero/a; Fisioterapeuta; Auxiliar de Enfermería; Veterinario/a; Farmacéutico/a.

Finalmente los estudiantes proceden a valorar las diecinueve áreas vocacionales recogidas en el CIBAP, de acuerdo con la siguiente situación estimular: Señala tu grado de PREFERENCIA E INTERÉS globalmente considerado hacia las distintas áreas vocacionales, de acuerdo con las siguientes categorías: 


\begin{tabular}{|c|c|c|c|c|}
\hline A & B & C & D & E \\
\hline La rechazo & No me gusta & $\begin{array}{c}\text { Me es indiferente } \\
\text { o tengo dudas }\end{array}$ & $\begin{array}{c}\text { Me gusta } \\
\text { Bastante }\end{array}$ & $\begin{array}{c}\text { Es de mis } \\
\text { preferidas }\end{array}$ \\
\hline
\end{tabular}

La versión definitiva empleada en nuestra investigación, aparece recogida en el ANEXO 1. A partir de una presentación inicial motivadora, acompañada de las explicaciones pertinentes y aclaración de dudas del Orientador antes de su aplicación, al final de la presentación del cuestionario, leemos la siguiente orientación: "El CIBAP te ayudará a clarificar los estudios y profesiones que más te gustan y te interesan. Responde con sinceridad, teniendo presente tus deseos y experiencias hasta el momento. De esta manera, los resultados que obtengas podrán ayudarte mejor a considerar tus próximas decisiones vocacionales”.

Como sabemos (Castaño, 1983: 210), las escalas de los cuestionarios de intereses pueden ser ipsativas y normativas. Se denominan ipsativas aquellas escalas en los que el perfil del individuo se evalúa en relación con su propio promedio o "baremo interno" sin que sea necesario efectuar ninguna comparación con algún grupo normativo o "baremo externo". Los perfiles a partir de estas puntuaciones nos muestran las preferencias relativas de unas áreas de interés respecto a otras. Las escalas normativas por otro lado, son aquellas en las que las puntuaciones de cada sujeto se interpretan en relación con las obtenidas por un grupo de referencia (grupo normativo). La puntuación de cada sujeto se interpreta, pues, en relación con las diferencias interindividuales, es decir, las obtenidas entre sus resultados y los del grupo normativo que sirve de referencia para la comparación.

El CIBAP en función de sus objetivos lo hemos concebido como un cuestionario para ser interpretado ipsativamente, quedando abierta la posibilidad de explorar para más adelante la elaboración de baremos, construidos a partir de perfiles empíricos procedentes de grupos normativos que pueden hacer referencia a distintas carreras o profesiones.

Hemos decantado nuestra opción metodológica por las puntuaciones ipsativas, pues sobre la base del objetivo de esta investigación, pretendemos que a partir de la información vocacional facilitada por el CIBAP sobre el alcance de cada una de las áreas vocacionales consideradas, el alumno identifique su área vocacional de interés preferente. Esta elección por parte del estudiante, es un proceso principalmente subjetivo y mediado por un conjunto de cogniciones vocacionales cuyo estudio nos proponemos abordar en trabajos posteriores.

\section{Muestra}

Hemos contado para la realización de este estudio con una muestra incidental de carácter no-probabilística de alumnos del cuarto curso de secundaria y del segundo curso de los nuevos bachilleratos LOGSE. La muestra que contestó a la totalidad de los cuestionarios y que, además, lo hizo correctamente ("muestra productora de datos"; Fox, 1981), procede de distintos ámbitos geográficos y tipos de centros.

La muestra está compuesta por un total de 1.250 estudiantes. Todos ellos proceden de cuatro centros públicos de Enseñanza Secundaria (IES), tres concertados en ESO, Bachilleratos y Ciclos Formativos (antiguos centros de FP) y tres privados concertados en las etapas obligatorias, tradicionalmente considerados como de BUP-COU y con oferta no concertada 
de Bachilleratos LOGSE, en total diez centros. Nos pareció importante de cara a una mayor representatividad de la muestra, además de los IES, incluir a centros de iniciativa social, diferenciando los que son privados con alguna etapa concertada, tradicionalmente considerados como de BUP-COU, de los también concertados pero provenientes de la antigua FP, por atender poblaciones de características muy diferentes desde el punto de vista del estatus sociofamiliar y que pueden afectar a los intereses y valores profesionales de los estudiantes, objeto de nuestro trabajo.

Del total de la muestra 919 estudiantes $(73,5 \%)$ estaban cursando $4^{\circ}$ de ESO y los 331 restantes $(26,5 \%)$ cursaban el segundo curso de Bachillerato en las distintas modalidades recogidas en la LOGSE. Por último el 58,6\% (732) son chicos y el 41,4\% restante son chicas (518).

\section{Recogida de datos}

Es muy importante destacar cuando consideramos la fiabilidad y validez de los datos obtenidos en este trabajo, que la aplicación cualificada de los cuestionarios se efectúo por los propios Orientadores de los Centros colaboradores en esta investigación, indicando, expresamente, que las respuestas no tienen consecuencias sobre las calificaciones escolares y solicitando la máxima sinceridad. Conseguimos un óptimo clima de colaboración de los estudiantes en la realización de los cuestionarios, al ser presentados en el marco del propio programa de orientación del centro como una actividad significativa de reflexión sobre su perfil de intereses vocacionales y un instrumento de ayuda de cara a su elección de continuidad de estudios al año siguiente. El cuestionario se les presento en forma de cuadernillo de actividades autoaplicable, de los que están acostumbrados a realizar en las sesiones del programa de Tutoría y Orientación.

\section{Análisis}

En primer termino presentamos un análisis descriptivo de la distribución de las áreas vocacionales de interés preferente de los estudiantes de secundaria en relación con las variable de etapa, grado de decisión y expectativa vocacional de logro. Exploramos las relaciones de interdependencia entre las mismas a través de pruebas de ji-cuadrado y mediante análisis de varianza el efecto del grado de decisión sobre las expectativas vocacionales de logro. Por último, comprobamos mediante la prueba t de Student de diferencias de medias para grupos independientes, la existencia de diferencias significativas en las áreas vocacionales de intereses básicos de los estudiantes de secundaria, en relación con la variable de etapa.

Todos los participantes en esta investigación y la información obtenida han sido tratados de acuerdo con los principios éticos para la investigación científica. Los análisis estadísticos se han realizado principalmente con los programas EXCEL, SPSS y EQS.

\section{Resultados y discusión}

Considerando el objetivo general que hemos indicado anteriormente y los objetivos específicos enunciados, vamos a exponer los resultados de nuestro trabajo en relación con las hipótesis formuladas. 


\section{Hipótesis primera}

La información facilitada por el CIBAP permite a los estudiantes de secundaria a partir de su perfil de intereses expresados, identificar su "área vocacional de interés preferente".

La preferencia por un área vocacional va más allá del mero interés y supone el resultado de un proceso cognitivo de elección, resultado de la comparación entre distintas opciones. En esencia recoge la afirmación: "Yo quiero ser X". (Rivas, F. 1998: 70) Una vez cumplimentado el CIBAP, se propuso a los estudiantes la siguiente situación estimular: "Revisa nuevamente las áreas vocacionales del Cuestionario de Intereses Académico Profesionales. Decide de entre los diecinueve grupos cual es tu área de interés preferente, esto es, aquella donde tu encuentres una profesión o estudios que te gustaría realizar en un futuro próximo.

Como conclusión sustantiva y en línea con lo postulado por la hipótesis primera, hemos comprobado afirmativamente que todos los estudiantes, tras la información sobre el contenido de cada una de las Áreas de Intereses Básicos recogidas en el CIBAP, han elegido de entre las diecinueve propuestas su "Área Vocacional Preferente". Asimismo y en lo que respecta a la distribución total y en función de su etapa del área vocacional de interés preferente de los estudiantes de la muestra, aparece recogida en la tabla 1.

Los resultados de las pruebas de contingencia nos informan que existe una relación de dependencia significativa entre el área vocacional de interés preferente de los estudiantes de la muestra y la variable etapa (Chi-cuadrado de Pearson $=44,138 ;$ g.l. $=18 ; \mathrm{p}=, 001$; $\mathrm{C}=, 185)$. Es decir, la distribución de las áreas vocacionales de interés preferente de los estudiantes de secundaria entre las diecinueve áreas posibles esta afectada por la etapa educativa donde se encuentran, y es significativamente diferente en cuarto de ESO en relación con segundo de Bachillerato.

Observamos que en la muestra total, en el ranking de las áreas vocacionales de interés preferente, el primer lugar lo ocupa el área de Investigación Técnica $(15,4 \%)$ seguida de Sanidad $(13,1 \%)$ y en tercer lugar Economía y Negocios $(10,4 \%)$. Este orden se corresponde tanto con la submuestra de cuarto de ESO como con la de segundo de Bachillerato, excepto en la tercera posición que en Bachillerato es ocupada por el área vocacional de Enseñanza y Orientación $(11,8 \%)$ que desplaza a Economía y Negocios $(8,5 \%)$ a la cuarta posición. Aunque la comparación de los resultados parciales de las áreas vocacionales de los estudiantes de secundaria en relación con su etapa nos ofrece grandes similaridades que nos permiten conjeturar que al finalizar la ESO los intereses profesionales se encuentran ya "cristalizados" y van a permanecer estables a lo largo del bachillerato, algunas diferencias como las encontradas en las áreas de Enseñanza y de Derecho nos parecen sugerentes. Al pertenecer las dos submuestras a cohortes diferentes, para comprobar la hipótesis de la estabilidad de los intereses sería necesario abordar un estudio longitudinal de la submuestra de $4^{\circ}$ de ESO y volverla a estudiar cuando accediese a $2^{\circ}$ de bachillerato.

\section{Hipótesis segunda}

Los intereses de los estudiantes tanto de cuarto de ESO como los de segundo de Bachillerato se encuentran definidos entre una y tres áreas vocacionales y es muy bajo el porcentaje de estudiantes indefinidos vocacionalmente en estos cursos. 


\section{TABLA 1: Distribución de las Áreas Vocacionales Preferentes en relación con la "etapa".}

\begin{tabular}{|c|c|c|c|c|c|c|c|c|}
\hline \multirow{3}{*}{$\begin{array}{l}\text { ÁREA VOCACIONAL } \\
\text { DEINTERES } \\
\text { PREFERENTE }\end{array}$} & \multicolumn{8}{|c|}{ DISTRIBUCIÓN DE FRECUENCIAS } \\
\hline & \multicolumn{2}{|c|}{$4^{\circ}$ ESO } & \multicolumn{2}{|c|}{$2^{\circ}$ BTO } & \multicolumn{2}{|c|}{ TOTAL } & \multicolumn{2}{|c|}{$\begin{array}{c}\text { Área Única } \\
\text { sobre el total }\end{array}$} \\
\hline & $\mathbf{N}$ & $\%$ & $\mathbf{N}$ & $\%$ & $\mathbf{N}$ & $\%$ & $\mathbf{N}$ & $\%$ \\
\hline 1. Investigación cientifica & 50 & 5,4 & 18 & 5,4 & 68 & 5,4 & 18 & 26,5 \\
\hline 2. Investigación técnica & 140 & $15,2_{(1)}$ & 52 & $15,7_{(1)}$ & 192 & $15,4_{(1)}$ & 48 & 25,0 \\
\hline 3. Tecnica aplicada & 70 & 7,6 & 26 & 7,9 & 96 & 7,7 & 24 & 25,0 \\
\hline 4. Sanidad & 119 & $12,9_{(2)}$ & 45 & $13,6_{(2)}$ & 164 & $13,1_{(2)}$ & 59 & $36,0_{(4)}$ \\
\hline 5. Economia y negocios & 102 & $11,1_{(3)}$ & 28 & $8,5_{(4)}$ & 130 & $10,4_{(3)}$ & 25 & 19,2 \\
\hline 6. Actividades administrativas & 40 & 4,4 & 11 & 3,3 & 51 & 4,1 & 11 & 21,6 \\
\hline 7. Derecho y asesoramiento & 47 & 5,1 & 5 & 1,5 & 52 & 4,2 & 16 & 30,8 \\
\hline 8. Protección y seguridad & 52 & 5,7 & 20 & 6,0 & 72 & 5,8 & 27 & $37,5_{(3)}$ \\
\hline 9. Humanístico-social & 22 & 2,4 & 9 & 2,7 & 31 & 2,5 & 10 & 32,3 \\
\hline 10. Comunicación & 66 & 7,2 & 21 & 6,3 & 87 & 7,0 & 21 & 24,1 \\
\hline 11. Enseñanza y orientación & 50 & 5,4 & 39 & $11,8_{(3)}$ & 89 & 7,1 & 27 & 30,3 \\
\hline 12. Atención personal & 15 & 1,6 & 15 & 4,5 & 30 & 2,4 & 5 & 16,7 \\
\hline 13. Idiomás & 20 & 2,2 & 4 & 1,2 & 24 & 1,9 & 8 & $33,3_{(5)}$ \\
\hline 14. Cine y teatro & 20 & 2,2 & 4 & 1,2 & 24 & 1,9 & 1 & 4,2 \\
\hline 15. Música & 6 & ,7 & 5 & 1,5 & 11 & ,9 & 5 & $45,5_{(1)}$ \\
\hline 16. Artes plásticas & 23 & 2,5 & 5 & 1,5 & 28 & 2,2 & 3 & 10,7 \\
\hline 17. Estética & 16 & 1,7 & 1 & ,3 & 17 & 1,4 & 2 & 11,8 \\
\hline 18. Actividad agropecuaria & 14 & 1,5 & 2 & ,6 & 16 & 1,3 & 7 & $43,8_{(2)}$ \\
\hline 19. Deporte & 47 & 5,1 & 21 & 6,3 & 68 & 5,4 & 21 & 30,9 \\
\hline TOTAL & 919 & 100,0 & 331 & 100,0 & 1250 & 100,0 & 338 & 27,0 \\
\hline
\end{tabular}

( ) Entre paréntesis posición de cada área vocacional en un Ranking en función del porcentaje de estudiantes que la eligen.

Para estimar la certidumbre en la elección que hemos denominado "grado de decisión" nos preguntamos ¿El área vocacional elegida es su única de interés preferente o refleja su opción obligada entre varias? Para dar respuesta a este apartado les interrogamos sobre la siguiente cuestión: "De acuerdo con tus respuestas dadas en el CIBAP, tu área vocacional de interés preferente elegida se encuentra”: a) Entre las que te son indiferentes; b) Entre las que te gustan bastante; c) Entre tus tres o más preferidas; d) Entre tus dos preferidas; e) Es tu única preferida. Entendemos que los estudiantes situados en esta última categoría 
"única preferida" son los que presentan un mayor grado de certidumbre en la elección de su área vocacional de interés preferente, pues los grupos de estudiantes de las otras categorías tienen repartido su interés entre varias, aunque el proceso de elección les lleve a tener que pronunciarse sólo por una renunciando a otras. En el otro extremo de la escala, situaríamos a los estudiantes indecisos que reparten su interés entre una o varias áreas que les gustan bastante, pero aún no encuentran ninguna área que sea de sus preferidas.

Encontramos que el total de la muestra presenta claramente unos perfiles de intereses diferenciados, así un $27 \%$ sitúan su área vocacional de interés preferente como la "única preferida", dos preferidas el $38,1 \%$, tres el $26,2 \%$ y sólo el $8,7 \%$ no tienen un área preferida y presentan aún un alto grado de indefinición de sus intereses. La distribución de la submuestra de sujetos de "área única" se puede comprobar también en la tabla 4. Destacaríamos el dato del área de Sanidad que ocupa el segundo puesto en el ranking de áreas de interés y en la que el $36,0 \%$ de los sujetos manifiestan que es su única área de interés preferente.

\section{Hipótesis tercera}

No existen diferencias significativas en el grado de decisión vocacional de los estudiantes de cuarto de ESO con relación a los de segundo de Bachillerato.

¿Podemos esperar que los estudiantes de $2^{\circ}$ de bachillerato tengan mas definida su "única área preferida" que los estudiantes de $4^{\circ}$ de ESO, teniendo en cuenta su mayor formación y la inmediatez de su elección de continuidad de estudios universitarios o profesionales? Por tanto ¿Existen diferencias en el "grado de decisión" en función de la etapa de los estudiantes? La distribución de frecuencias de las categorías de la variable "grado de decisión” en relación con la etapa aparecen recogidas en la tabla 2.

Los resultados de las pruebas de contingencia nos informan que no existe una relación de dependencia significativa entre el "grado de decisión" del área vocacional de interés preferente de los estudiantes de la muestra en función de su etapa (Chi-cuadrado de Pearson $=5,915 ;$ g.l. $=3 ; \mathrm{p}<, 116$ ). Por tanto, el "grado de decisión" del área vocacional de in-

\section{TABLA 2: Distribución del grado de decisión de las Áreas Vocacionales Preferentes en relación con la "etapa".}

\begin{tabular}{|c|c|c|c|c|c|c|}
\hline \multirow[b]{2}{*}{ Grado de decisión } & \multicolumn{6}{|c|}{ DISTRIBUCIÓN DE FRECUENCIAS } \\
\hline & $\begin{array}{c}\text { N } \\
4^{\circ} \text { ESO }\end{array}$ & $\begin{array}{c}\% \\
4^{\circ} \stackrel{\text { ESO }}{ }\end{array}$ & $\begin{array}{ll} & \mathrm{N} \\
2^{\circ} & \text { BTO }\end{array}$ & $\begin{array}{c}\% \\
2^{\circ} \text { BTO }\end{array}$ & $\begin{array}{c}\mathbf{N} \\
\text { TOTAL }\end{array}$ & $\begin{array}{c}\% \\
\text { TOTAL }\end{array}$ \\
\hline Única preferida & 241 & 26,2 & 97 & 29,3 & 338 & 27,0 \\
\hline Dos preferidas & 343 & 37,3 & 133 & 40,2 & 476 & 38,1 \\
\hline Tres preferidas & 257 & 28,0 & 70 & 21,1 & 327 & 26,2 \\
\hline Las que me gustan bastante & 78 & 8,5 & 31 & 9,4 & 109 & 8,7 \\
\hline Las que me son indiferentes & 0 & 0 & 0 & 0 & 0 & 0 \\
\hline
\end{tabular}


Evaluación de los intereses básicos académico...

terés preferente de los estudiantes de secundaria no esta afectado significativamente por la variable etapa.

\section{Hipótesis cuarta}

Los estudiantes de secundaria presentan unas altas expectativas de logro hacia su área vocacional de interés preferente.

Una vez conocida la distribución de las áreas de intereses en estas etapas nos planteamos el siguiente interrogante $¿$ Qué expectativa tienen los estudiantes de secundaria de poder realizar finalmente alguno de los estudios o actividades profesionales de su interés perteneciente a su área vocacional preferente elegida? Entendemos que dicha creencia se corresponde con lo que hemos denominado "expectativas vocacionales de logro" de los estudiantes de secundaria. Las categorías consideradas para la variable expectativas vocacionales de logro fueron las siguientes: a) Pienso que nunca llegaré a desempeñarla; b) Creo que tengo muy pocas posibilidades; c) Pienso que es probable que pueda realizarla; y d) Tengo una gran seguridad en conseguirlo.

Como podemos observar en la tabla 3, las "expectativas vocacionales de logro" son muy altas tanto en los estudiantes de cuarto de ESO como en los de segundo de Bachillerato. Estos resultados nos permiten afirmar que la mayor proporción de los estudiantes de estas etapas $(92,8 \%)$, guiados por su conocimiento de la realidad socioprofesional, manifiestan interés hacia aquellas áreas vocacionales donde perciben que tienen probabilidades de conseguir lo que desean, siendo muy pocos ya los que a estas edades, llevados por sus fantasías, expresan su interés hacia áreas en las que anticipan que tienen muy pocas o nulas posibilidades de alcanzar lo que desean (7,2\%). En conclusión podemos afirmar que les interesa aquello que anticipan que creen tener probabilidades de llegar a conseguir.

\section{TABLA 3: Distribución del grado de decisión de las Areas Vocacionales de Interés Preferente evaluadas por los estudiantes en relación con la "etapa".}

\begin{tabular}{|l|c|c|c|c|c|c|}
\hline \multirow{2}{*}{$\begin{array}{c}\text { Expectativas vocacionales } \\
\text { de logro }\end{array}$} & \multicolumn{6}{|c|}{ DISTRIBUCIÓN DE FRECUENCIAS EN CADA ETAPA } \\
\cline { 2 - 7 } & $\begin{array}{c}\mathbf{N} \\
\mathbf{4}^{\mathbf{0}} \text { ESO }\end{array}$ & $\begin{array}{c}\mathbf{\%} \\
\mathbf{4}^{\mathbf{0}} \text { ESO }\end{array}$ & $\begin{array}{c}\mathbf{N} \\
\mathbf{2}^{\mathbf{0}} \text { BTO }\end{array}$ & $\begin{array}{c}\mathbf{\%} \\
\mathbf{2}^{\mathbf{0}} \mathbf{B T O}\end{array}$ & $\begin{array}{c}\mathbf{N} \\
\text { TOTAL }\end{array}$ & $\begin{array}{c}\mathbf{\%} \\
\text { TOTAL }\end{array}$ \\
\hline Nunca llegaré a desempeñarla & 8 &, 9 & 2 &, 6 & 10 &, 8 \\
\hline Tengo muy pocas posibilidades & 53 & 5,9 & 26 & 8,0 & 79 & 6,4 \\
\hline Probable que pueda realizarla & 516 & 57,2 & 199 & 61,2 & 715 & 58,3 \\
\hline Gran seguridad en conseguirlo & 325 & 36,0 & 98 & 30,2 & 423 & 34,5 \\
\hline
\end{tabular}

\section{Hipótesis quinta}

Los estudiantes de secundaria más determinados en su área vocacional de interés preferente, están significativamente más seguros de llegar a desempeñar los estudios o profesión de su interés que los pertenecientes al grupo de estudiantes indecisos. 
A continuación vamos a estudiar el efecto del "grado de decisión" del área vocacional de interés preferente de los estudiantes de secundaria sobre sus "expectativas vocacionales de logro". Encontramos que los resultados de las pruebas de contingencia nos muestran que existe una relación de dependencia significativa entre las variables "grado de decisión" del área vocacional de interés preferente y "expectativa vocacional de logro" (Chi-cuadrado de Pearson $=29,125 ;$ g.l. $=9 ; \mathrm{p}=, 001 ; \mathrm{C}=, 151)$. Por ello es plausible conjeturar que el grado de decisión hacia el área vocacional de interés preferente esté afectando las expectativas vocacionales de logro de los estudiantes. En este sentido podemos esperar que los estudiantes que tienen más definidos sus intereses profesionales, presenten mayores expectativas vocacionales de logro que los que se muestran más indecisos. Seguidamente, comprobamos a través del análisis de varianza la existencia de probabilidades asociadas entre ambas variables y obtenemos los resultados que se recogen en la tabla 4.

TABLA 4: Análisis de varianza entre el grado de decisión vocacional de los estudiantes en función de sus expectativas vocacionales de logro.

\begin{tabular}{|c|c|c|c|c|c|c|c|}
\hline $\begin{array}{c}\text { Variable } \\
\text { dependiente }\end{array}$ & $\begin{array}{l}\text { Grado de decisión } \\
\text { vocacional }\end{array}$ & $\mathbf{N}$ & Media* & G.L. & $\mathbf{F}$ & Sig. & $\eta^{2}$ \\
\hline \multirow{5}{*}{$\begin{array}{c}\text { Expectativas } \\
\text { vocacionales } \\
\text { de logro }\end{array}$} & 1. Las que me gustan bastante & 108 & $3,10_{(4)}$ & \multirow{5}{*}{$\begin{array}{r}3 \\
1223 \\
1226\end{array}$} & \multirow{5}{*}{5,980} & \multirow{5}{*}{, 000} & \multirow{5}{*}{, 0146} \\
\hline & 2. Tres preferida & 324 & 3,27 & & & & \\
\hline & 3. Dos preferidas & 460 & $3,23_{(4)}$ & & & & \\
\hline & 4. Única preferida & 335 & $3,36_{(1)(3)}$ & & & & \\
\hline & Total & 1227 & 3,26 & & & & \\
\hline
\end{tabular}

* Entre paréntesis se encuentra el grupo con el cual presenta diferencias significativas; prueba post hoc de Scheffé $(\alpha=, 05)$

Como se aprecia en la tabla, el análisis de varianza entre estas dos variables nos presenta que existen diferencias estadísticamente significativas $(\mathrm{p}<, 001)$ en las expectativas vocacionales de logro de los estudiantes de secundaria en relación con su grado de decisión vocacional. Si contemplamos la significatividad de las diferencias de medias entre los grupos en la variable "expectativas vocacionales de logro" en función de las categorías de la variable "grado de decisión vocacional", podemos afirmar tal como establecimos en nuestra hipótesis que los estudiantes más determinados en su área vocacional de interés preferente, están significativamente más seguros de llegar a desempeñar los estudios o profesión de su interés que los pertenecientes al grupo de estudiantes indecisos.

\section{Hipótesis sexta}

Existen diferencias significativas en las valoraciones de las áreas vocacionales de intereses básicos de los estudiantes de secundaria en relación con la variable de etapa.

Por lo que concierne al análisis de las diferencias entre las valoraciones de las áreas vocacionales de intereses básicos de los estudiantes de secundaria en relación con la etapa que están cursando, comprobamos a través de la prueba $t$ de Student de diferencias de medias para 
grupos independientes, que existen probabilidades asociadas entre los intereses profesionales y la etapa. Como se aprecia en la tabla 5, solamente encontramos diferencias estadísticamente significativas en cuatro de las diecinueve áreas del CIBAP en relación con la etapa de los estudiantes de secundaria. En las demás áreas las diferencias de medias no son significativas.

TABLA 5: Prueba t de Student para las diferencias en los intereses profesionales de los estudiantes en función de la etapa educativa.

\begin{tabular}{|c|c|c|c|c|c|c|c|c|}
\hline Áreas CIBAP & Etapa & $\mathbf{N}$ & Media & Desv & $t$ & g.l. & Sig. & d \\
\hline \multirow{3}{*}{ ECONOMIA } & $4^{\circ} \mathrm{ESO}$ & 915 & 3,16 & 1,223 & \multirow{3}{*}{3,491} & \multirow{3}{*}{590,282} & \multirow{3}{*}{, 001} & \multirow{3}{*}{, 183 } \\
\hline & $2^{\circ} \mathrm{BTO}$ & 330 & 2,89 & 1,203 & & & & \\
\hline & Total & 1245 & 3,09 & 1,22 & & & & \\
\hline \multirow{3}{*}{ DERECHO } & $4^{\circ} \mathrm{ESO}$ & 918 & 2,65 & 1,232 & \multirow{3}{*}{5,466} & \multirow{3}{*}{670,239} & \multirow{3}{*}{, 000} & \multirow{3}{*}{,328 } \\
\hline & $2^{\circ} \mathrm{BTO}$ & 331 & 2,26 & 1,063 & & & & \\
\hline & Total & 1249 & 2,54 & 1,20 & & & & \\
\hline \multirow{3}{*}{ ENSEÑANZA } & $4^{\circ} \mathrm{ESO}$ & 917 & 2,87 & 1,180 & \multirow{3}{*}{$-5,664$} & \multirow{3}{*}{600,884} & \multirow{3}{*}{, 000} & \multirow{3}{*}{,359 } \\
\hline & $2^{\circ} \mathrm{BTO}$ & 331 & 3,29 & 1,142 & & & & \\
\hline & Total & 1248 & 2,99 & 1,18 & & & & \\
\hline \multirow{3}{*}{$\begin{array}{l}\text { ATENCIÓN } \\
\text { PERSONAL }\end{array}$} & $4^{\circ} \mathrm{ESO}$ & 917 & 2,68 & 1,044 & \multirow{3}{*}{$-5,210$} & \multirow{3}{*}{565,346} & \multirow{3}{*}{, 000} & \multirow{3}{*}{, 341} \\
\hline & $2^{\circ} \mathrm{BTO}$ & 331 & 3,04 & 1,083 & & & & \\
\hline & Total & 1248 & 2,78 & 1,06 & & & & \\
\hline
\end{tabular}

El examen de los resultados nos muestra que de las diecinueve áreas vocacionales recogidas en el CIBAP sólo encontramos diferencias significativas en cuatro áreas en relación con la variable etapa. Este dato nos esta indicando un detalle de extraordinario valor desde la perspectiva de la eficacia de los programas de orientación: la distribución de los intereses profesionales de los estudiantes de cuarto de secundaria es muy semejante a la de sus compañeros de segundo de bachillerato, en concreto en quince áreas vocacionales. Este análisis ofrece indicios empíricos a la hipótesis de la estabilidad de la distribución de los intereses profesionales de los estudiantes de cuarto de secundaria con respecto a sus futuros intereses dos años después al terminar el bachillerato, aunque como ya hemos señalado es necesario realizar un estudio longitudinal para validar empíricamente esta hipótesis.

Por otra parte, como podemos observar el interés por el área vocacional de Economía por un lado y de Derecho por otro, es significativamente mayor en los alumnos de cuarto de secundaria que en los alumnos de segundo de bachillerato. ¿Por qué? Una posible explicación guarda relación con el efecto moderador del rendimiento académico de los estudiantes con interés por estas áreas en función de la etapa. El proceso sería el siguiente: como consecuencia de los malos resultados académicos, se produce la eliminación antes de llegar a $2^{\circ}$ de Bachillerato de una parte de los alumnos que estaban interesados en $4^{\circ}$ de ESO por estas áreas, tanto por sus dificultades en la obtención del título de graduado en secundaria para el acceso al Bachillerato como en su finalización con éxito. 
Por último, encontramos que el interés por el área vocacional de Enseñanza por un lado y de Atención Personal por otro, es significativamente mayor en los alumnos de segundo de Bachillerato que en los alumnos de cuarto de ESO. ¿Por qué? Nuestra hipótesis explicativa se orienta a afirmar que el interés por las profesiones relacionadas con la Enseñanza y la Atención Personal tiene un primer periodo de cristalización hacia el final de la adolescencia en cuarto de ESO. Asimismo, hacia el final del Bachillerato y probablemente movidos por cambios en sus sistemas de valores, sobreviene una nueva eclosión en los jóvenes hacia el interés por estas áreas.

\section{Conclusiones}

De lo expuesto en nuestro trabajo podemos concluir que el CIBAP cumple satisfactoriamente una función informativa en el ámbito de la exploración de carrera y a partir de esta información facilita al estudiante identificar su "Área Vocacional Preferente". Su aplicación en el marco de los programas de orientación al final de la Enseñanza Secundaria Obligatoria y los Bachilleratos, ha puesto de manifiesto en los diez centros participantes en este estudio, que nos permite ayudar a los estudiantes a comprender mejor el contenido y diferenciación de las principales Áreas Vocacionales existentes y tomando estas como punto de partida, elaborar a partir de sus puntuaciones ipsativas, un perfil descriptivo de sus Intereses Profesionales Básicos. En este perfil podemos ver plasmada a pesar de su complejidad, su autoconciencia sobre sus preferencias y rechazos en términos vocacionales, como paso previo a la elección de continuidad de estudios académicos o profesionales a la que se enfrenta en estos cursos críticos, de transición de una etapa educativa a otra.

Todos los estudiantes, tras la información sobre el contenido de cada una de las Áreas de Intereses Básicos recogidas en el CIBAP, han decidido su "Área Vocacional Preferente". Hemos verificado su distribución entre las diecinueve áreas que explora este instrumento, analizando su "grado de decisión" hacia las mismas y las expectativas de acceder finalmente a unos estudios o profesión de su interés de los incluidos en su área vocacional de interés preferente. En resumen, de acuerdo con lo postulado en las hipótesis enunciadas en nuestro trabajo, hemos comprobado que:

- La información facilitada por el CIBAP permite a los estudiantes de secundaria a partir de su perfil de intereses expresados, identificar su "área vocacional de interés preferente".

- Los intereses de los estudiantes tanto de cuarto de ESO como los de segundo de Bachillerato se encuentran definidos entre una y tres áreas vocacionales y es muy bajo el porcentaje de estudiantes que no están definidos vocacionalmente en estos cursos.

- No existen diferencias significativas en el grado de decisión vocacional de los estudiantes de cuarto de ESO con relación a los de segundo de Bachillerato.

- Los estudiantes de secundaria presentan unas altas expectativas de logro hacia su área vocacional de interés preferente.

- Los estudiantes de secundaria más determinados en su área vocacional de interés preferente, están significativamente más seguros de llegar a desempeñar los estudios o profesión de su interés que los pertenecientes al grupo de estudiantes indecisos. 
Por último, como conclusión final y más sustantiva, después de los datos recogidos en los diferentes análisis realizados, hemos comprobado que los intereses profesionales en cuarto de ESO son semejantes a los de los estudiantes de segundo de Bachillerato en quince de las diecinueve áreas vocacionales recogidas en el CIBAP. Esta semejanza la podemos interpretar como un indicador de la estabilidad de la distribución de los intereses al finalizar la ESO en relación con los encontrados en segundo de bachillerato, aunque para validar empíricamente esta hipótesis necesitamos por una lado controlar la varianza debida a las diferencias de cohorte de nuestras submuestras mediante un estudio longitudinal y por otro estudiar el efecto de otras variables moderadoras sobre los intereses no contempladas en este trabajo como el rendimiento académico de los estudiantes, su estatus sociofamiliar, el centro educativo y el género que pensamos abordar en futuras publicaciones sobre esta línea de investigación.

De todo lo expuesto en nuestro trabajo, queremos resaltar que es obligado considerar la evaluación de los intereses profesionales como parte esencial de los procesos de orientación tanto por su utilidad para conocer la atracción actual que tiene un individuo por algún campo vocacional como paso previo a la elección de estudios o profesión como para facilitar en el individuo el desarrollo de sus habilidades para la toma de decisiones vocacionales y confiamos que este estudio haya contribuido a clarificar y actualizar nuestro conocimiento sobre este importante tema.

\section{Referencias bibliográficas}

Apostal, R. A. (1985). "Expressed-inventoried interest agreement and type of Strong- Campbell Interest Inventory Scale". Journal of Counseling Psychology, 32, 634-636.

Athanasou, J. A., \& Cooksey, R. W (1993). "Self-estimates of vocational interest". Australian Psychologist, 28, 118-127.

Bartling, H. C., \& Hood, A. B. (1981). "An 11-year follow-up of measured interest and vocational choice". Journal of Counseling Psychology, 28, 27-35.

Betsworth, D. G. \& Fouad, N. A. (1997). "Vocational Interest: A look at the past 70 years and a glance at the future". The Career Development Quarterly, 46, 23-47.

Borgen, F. H. (1986). "New approaches to the assessment of interests". In W. B. Walsh \& S. H. Osipow (Eds.), Advances in vocational psychology: Vol. 1 The assessment of interests (pp. 31-54). Hillsdale, NJ: Erlbaum.

Borgen, F. H., \& Seling, M. J. (1978). "Expressed and inventoried interests revisited: perspicacity in the person". Journal of Counseling Psychology, 25, 536-543.

Castaño López-Mesas, C. (1983). Psicología y orientación vocacional. Madrid: Marova.

Darley, J. G., \& Hagenah, T. (1955). Vocational interest measurement: Theory and practice. Minneapolis: University of Minnesota Press.

Day, S. X., \& Rounds, J. (1997). "A little more than kind, and less than kind: basic interest in vocational research and career counseling". The Career Development Quarterly, 45, 3, 207-220.

De La Cruz López, Ma. V. (1997). IPP Inventario de Intereses y Preferencias Profesionales. Manual, $3^{\mathrm{a}}$ edición, revisada. Madrid: TEA Ediciones.

Dolliver, R. H. (1969). Strong Vocational Interest Blank versus expressed vocational interests: A review. Psychological Bulletin, 72, 95-107. 
Fryer, D. (1931). The measurement of interests. New York: Henry Holt.

Fox, D. (1981): El proceso de investigación en educación. Pamplona: EUNSA

García Mediavilla, L., Izquierdo, R. y Sánchez-Cabezudo, J. (1990). Test "CIP-II". Cómo descubrir los intereses profesionales. Madrid: Bruño.

Gelatt, H. B. (1962). "Decisions making: A conceptual framework for counselling". Journal of Counseling Psychology, 9, 240-245.

Gottfredson, L. S. (1986). "Special groups and the beneficial use of vocational interest inventories". In W. B. Walsh \& S. H. Osipow (Eds.), Advances in vocational psychology, Vol. 1: The assessment of interests (pp. 127-198). Hillsdale, NJ: Erlbaum.

Hansen, J. C. (1984a). "The measurement of vocational interests". In S. D. Brown \& R. W. Lent (Eds.), Handbook of counseling psychology (pp. 99-136), New York: Wiley.

Harmon, L. W., Hansen, J. C., Borgen, F. H., \& Hammer, A. L. (1994). Strong Interest Inventory applications and technical guide. Stanford, CA: Stanford University Press.

Hernández Franco, V. (2001). Análisis causal de los intereses profesionales en los estudiantes de secundaria. Tesis Doctoral en prensa. Madrid: Universidad Complutense.

Jordan, J. P. (1963). "Exploratory behaviour: The formation of self and occupational concepts". In D. E. Super (Ed.), Career development: Self-concept theory. New York: College Entrance Examination Board. 42-78.

Kuder G. F. (1939). Kuder Preference Record Personal, Form A. Chicago: University of Chicago Bookstore

Laing, J., Swaney, K., \& Prediger, D. J. (1984). "Integrating vocational interest inventory results and expressed choices". Journal of Vocational Behavior, 25, 304-315.

Morales Vallejo, P. (2000). Medición de actitudes en psicología y educación. $2^{a}$ ed. revisada. Madrid: Universidad Pontificia Comillas de Madrid.

Pascual, F. (1997). Caracterización vocacional del grupo Deportivo. Tesis de Licenciatura. Facultad de Psicología. Universidad de Valencia

Rivas Martínez, F. (1988). Psicología vocacional: Enfoques del asesoramiento. Madrid: Morata (2a ed., 1993)

Rivas Martínez, F et al. (1995). "Manual de asesoramiento y orientación vocacional”. Madrid: Síntesis.

Rivas Martínez, F. Rocabert, E. y López González, M. L. (1998). Manual del SAV-R y SAVI-2000. Sistemas de Autoayuda y Asesoramiento Vocacional. Valencia: Servicios de Asesoramiento Vocacional y Educativo, S.L.

Rocabert, E. (1987). La opción universitaria: un sistema de exploración de la conducta vocacional basado en los intereses y preferencias vocacionales, Tesis doctoral, Facultad de Psicología, Dpto. de Psicología Evolutiva y de la Educación, Universidad de Valencia.

Savickas, M. L. (1995). "Examining the personal meaning of inventoried interests during career counseling”. Journal of Career Assessment, 3, 188-201.

Strong, E. K. (1927). Vocational Interest Blank. Stanford, CA: Stanford University Press.

Strong, E. K. (1943). Vocational interests of men and women. Stanford, CA: Stanford University Press.

Super, D. E., \& Crites, J. 0. (1962). Appraising vocational fitness, 2a ed., New York: Harper y Row.

Tiedeman, D. V. (1961). Decisions and vocational development: A paradigm and its implications, Personnel and Guidance Journal, 40, 15-21. 
ANEXO 1

\section{CUESTIONARIO DE INTERESES BÁSICOS ACADÉMICO PROFESIONALES (CIBAP)}

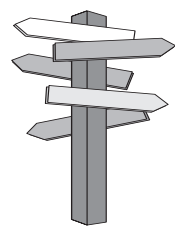

MIS INTERESES ACADÉMICO/PROFESIONALES

\begin{tabular}{|l|l|l|l|}
\hline \multicolumn{2}{|l|}{ Alumno } & Curso & $\mathrm{N}^{\circ}$ \\
\hline Sexo: $[\mathrm{V}][\mathrm{M}$ & Edad & Fecha \\
\cline { 3 - 4 }
\end{tabular}

Los intereses constituyen uno de los elementos más relevantes a tener en cuenta a la hora de tomar una decisión vocacional, señalan hacia donde dirige una persona sus deseos. Lo que nos interesa, nos gusta y atrae, es en cierta manera lo que motiva y dirige nuestra conducta; tener claro estos intereses, es muy importante de cara a tomar decisiones vocacionales satisfactorias.

Los intereses profesionales, lo que te gustaría estudiar o trabajar, se van conformando desde las experiencias escolares, familiares y del conocimiento que tengas del mundo laboral. En ese proceso, influyen muchos aspectos, unos proceden de las opiniones que tus padres, amigos y profesores hacen de tus proyectos; otros son menos visibles, como el que se recibe de los medios de comunicación, el cine, la situación laboral del país, etc. Pero todos tienen que pasar por tu criterio, reflexión o juicio sobre lo que de verdad quieres o piensas de tu futuro.

Este CUESTIONARIO DE INTERESES ACADEMICO PROFESIONALES (CIBAP) consta de diecinueve Áreas Vocacionales, sobre las que se te pide valorar tu grado de interés. Cada área de intereses agrupa un conjunto de estudios y profesiones que comparten aspectos comunes entre sí que les diferencian a su vez de las otras áreas. En cada grupo encontraras una breve descripción explicativa de sus características más relevantes y a continuación una breve referencia de algunos de los estudios universitarios representativos, así como la localización de las Familias Profesionales de ciclos formativos correspondientes, para terminar reseñando algunas profesiones afines a dichos estudios dentro de cada área vocacional.

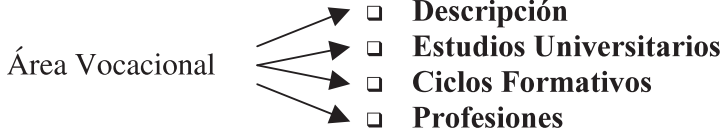

El CIBAP te ayudará a clarificar los estudios y profesiones que más te gustan y te interesan. Responde con sinceridad, teniendo presente tus deseos y experiencias hasta el momento. De esta manera, los resultados que obtengas podrán ayudarte mejor a considerar tus próximas decisiones vocacionales. 
Señala tu grado de PREFERENCIA E INTERÉS globalmente considerado por las diferentes áreas vocacionales, de acuerdo con la siguiente categorías:

\begin{tabular}{|c|c|c|c|c|}
\hline A & B & C & D & E \\
\hline Rechazo & No me gusta & $\begin{array}{c}\text { Me es indiferente } \\
\text { o tengo dudas }\end{array}$ & $\begin{array}{c}\text { Me gusta } \\
\text { Bastante }\end{array}$ & $\begin{array}{c}\text { Es de mis } \\
\text { preferidas }\end{array}$ \\
\hline
\end{tabular}

\section{Cuestión $\mathrm{n}^{\mathrm{o}}$ AREAS VOCACIONALES: Estudios y profesiones \\ ITEM 1 1. INVESTIGACIÓN CIENTÍFICA: \\ Interés por aprender, descubrir y demostrar nuevas teorías y técnicas dentro del campo de las ciencias. Tiene que ver con las tareas de laboratorio, la ex- perimentación, la comprobación de hipótesis, la interpretación y comproba- ción de los fenómenos de la Naturaleza. \\ Estudios universitarios: Matemáticas; Biología; Geología; Física; Química. Ciclos Formativos Familia Profesional de: Química. \\ Profesiones como: Metereólogo/a; Consultor/a de Investigación y Desarrollo (I+D); Astrónomo/a; Estadístico/a; Biólogo/a; Genetista; Auxiliar Técnico de Laboratorio.}

\section{ITEM 2 2. INVESTIGACIÓN TÉCNICA:}

Interés por profesiones relacionadas con estudios universitarios politécnicos desde la perspectiva del análisis y fundamentación teórica de las actividades tecnológicas.

Estudios universitarios: Arquitectura; Ingenierías Técnicas y Superiores en sus diferentes especialidades: Informática; Aeronáutica; Telecomunicaciones; Industrial; Caminos; Naval.

Profesiones como: Arquitecto/a; Ingeniero/a en sus diferentes especialidades; Programador/a Informático/a; Controlador/a Aéreo/a.

\section{ITEM 3 3. TÉCNICA APLICADA:}

Interés por profesiones relacionadas con el ámbito tecnológico e industrial. Tiene que ver con la creación y empleo de maquinaria, reparación o utilización de herramientas, trabajo manual, solución de problemas técnicos.

Ciclos Formativos Familias Profesionales de: Informática; Electricidad y Electrónica; Fabricación Mecánica; Edificación y Obra Civil; Madera y Mueble; Mantenimiento de Vehículos; Mantenimiento y Servicios a la Producción; Vidrio y Cerámica.

Profesiones como: Técnico Informático; Instalador Electricista; Técnico de reparaciones y mantenimiento electrónico; Carpintero/a; Fontanero/a; Mecánico/a (Avión, Barco, Automoción); Constructor; Operario de la Construcción 
(Albañil, Solador...) Delineante; Topógrafo/a; Transportista (Camionero, Taxista, Repartidor...); Piloto de Aviación Civil.

\section{ITEM 4 4. SANIDAD:}

Interés por profesiones relacionadas con la prevención, diágnostico, curación y conservación de la salud tanto física como mental de las personas o los animales.

Estudios universitarios: Medicina en alguna de sus diferentes especialidades; Psicología Clínica; Enfermería; Fisioterapia; Veterinaria; Farmacia.

Ciclos Formativos Familia Profesional de Sanidad.

Profesiones como: Medico de familia; Pediatra; Cirujano; Dentista; Psiquiatra; Enfermero/a; Fisioterapeuta; Auxiliar de enfermería; Veterinario/a; Farmacéutico/a.

\section{ITEM 5 5. ECONOMÍA Y NEGOCIOS:}

Interés por las profesiones relacionadas con actividades económicas, la administración de empresas y la labor comercial, bursátil y financiera... manejar y ser capaz de llevar negocios,.

Estudios universitarios: Administración y Dirección de Empresas; Economía; Investigación y Técnicas de Mercado (Marketing) Gestión y Administración Pública.

Profesiones como: Gerente; Director Comercial; Director de Contabilidad; Director de Marketing; Director de Banco; Auditor Financiero; Investigador de Mercados.

\section{ITEM 6 6. ACTIVIDADES ADMINISTRATIVAS}

Interés por profesiones relacionadas con el ámbito del sector servicios, caracterizadas por su componente administrativo, financiero y comercial de tipo práctico y aplicado. Se relaciona con la contabilidad, la administración, la gestión, los tramites burocráticos, etc.

Ciclos Formativos Familias Profesionales de: Administración y Gestión; Comercio y Marketing.

Profesiones como: Dependiente/a de comercio; Cajero/a; Contable, Interventor de Banca; Agente inmobiliario; Administrativo/a; Secretario/a; Agente Comercial; Telefonista.

\section{ITEM 7 7. DERECHO Y ASESORAMIENTO:}

Interés por las profesiones relacionadas con el funcionamiento de la sociedad, la legislación, la administración de justicia y la política. Tiene que ver con elaborar normas de convivencia y vigilar su cumplimiento, protegiendo a los ciudadanos de quienes no las obedecen

Estudios universitarios: Derecho; Ciencias Políticas y de la Administración; Relaciones laborales. 
Profesiones como: Abogado/a; Juez/a; Fiscal, Notario/a; Procurador/a; Asesor Fiscal; Consultor/a Jurídico/a; Jefe/a de Personal; Político/a; Diplomáti$\mathrm{co} / \mathrm{a}$

\section{ITEM 8 8. PROTECCIÓN Y SEGURIDAD:}

Interés por las profesiones dedicadas a la defensa y seguridad de las personas, relacionadas con el ámbito de los cuerpos y fuerzas de seguridad del Estado y autonómicos, administraciones locales y empresas privadas. Tiene que ver con disciplina y mando... dirigir y ordenar a personas que tienen puestos inferiores, así como aceptar y obedecer ordenes de una autoridad superior.

Profesiones como: Militar del Ejercito profesional en sus diferentes escalas y categorías; Policía Nacional, autonómica o local; Guardia Civil, Funcionario de Prisiones; Agente de Seguridad Privada; Técnico de Protección Civil; Bombero.

\section{ITEM 9 9. HUMANÍSTICO-SOCIAL:}

Interés por las profesiones relacionadas con la producción cultural, investigación y creación literarias... estudiar y conocer el pasado y presente del pensamiento y cultura de la humanidad.

Estudios universitarios: Geografía; Historia; Filosofía; Sociología; Antropología Social y Cultural; Humanidades; Biblioteconomía y Documentación. rofesiones como: Escritor/a; Historiador/a; Bibliotecario/a Documentalista; Sociólogo/a.

\section{ITEM 10 10. COMUNICACIÓN:}

Interés por las profesiones relacionadas con la información a través de los medios de comunicación social: prensa, radio y televisión y la creación de productos multimedia.

Estudios universitarios: Publicidad; Periodismo; Comunicación Audiovisual.

Ciclos Formativos Familia Profesional de: Imagen y Sonido.

Profesiones como: Redactor periodista; Locutor de radio; Presentador de TV.

\section{ITEM 11 11. ENSEÑANZA Y ORIENTACIÓN:}

Interés por las profesiones relacionadas con la educación, desarrollo, orientación y ayuda a otras personas.

Estudios universitarios: Pedagogía; Psicología educativa o Psicopedagogía; Magisterio; Educación Social; Trabajo Social.

Ciclos Formativos Familia Profesional de: Servicios Socioculturales y a la Comunidad.

Profesiones como: Orientador Escolar; Maestro de Primaria en las distintas especialidades o Profesor en Secundaria en las distintas áreas o materias; 
Técnico en Formación en la Empresa; Educador de Ocio y tiempo libre; Trabajador/a Social.

\section{ITEM 12 12. ATENCION PERSONAL:}

Interés por las profesiones relacionadas con servicios de atención y contacto con personas y grupos.

Estudios universitarios: Turismo.

Ciclos Formativos Familia Profesional de: Hostelería y Turismo.

Profesiones como: Gerente de Hotel; Agente de Viajes; Recepcionista; Conserje; Relaciones publicas; Animador/a Turístico; Guía Turístico.

\section{ITEM 13 13. IDIOMÁS:}

Interés por las profesiones relacionadas con el estudio y uso de la lengua y de otros idiomas.

Estudios universitarios: Traducción e Interpretación; Filología en sus distintas especialidades.

Profesiones como: Traductor; Interprete; Corresponsal periodista.

\section{ITEM 14 14.CINE Y TEATRO:}

Interés por las profesiones relacionadas con interpretación, creación artística en cine, teatro, televisión.

Estudios universitarios: Arte Dramático.

Profesiones como: Guionista; Actor; Cámara; Montador; Fotografía; Realizador; Doblaje.

\section{ITEM 15 15. MÚSICA:}

Interés por las profesiones relacionadas con composición, interpretación musical, vocal o corporal.

Estudios universitarios: Historia y Ciencias de la Música.

Estudios Profesionales: Grado elemental, medio y superior de Música o Danza en sus diferentes especialidades.

Profesiones como: Compositor; Instrumentista; Bailarín/a; Coreógrafo/a; Cantante.

\section{ITEM 16 16. ARTES PLÁSTICAS:}

Interés por las profesiones relacionadas con actividades artísticas, color y forma de objetos.... utilizar mi capacidad para manifestar lo que pienso, siento o quiero a través de actividades como la pintura, escultura, etc.

Estudios universitarios: Bellas Artes; Conservación y Restauración; Diseño.

Ciclos Formativos de: Artes Plásticas y Diseño; Artes Gráficas.

Profesiones como: Escenógrafo; Restaurador Artístico; Decorador/a; Dibujante; Escaparatista. 


\section{ITEM 17 17. ESTÉTICA: \\ Interés por las profesiones relacionadas con la atención y cuidado de la ima- gen corporal de las personas. \\ Ciclos Formativos Familias Profesionales de Imagen Personal y Textil. Profesiones como: Maquillador/a; Diseñador de modas; Modelo; Peluque- ro/a.}

\section{ITEM 18 18. ACTIVIDAD AGROPECUARIA:}

Interés por profesiones relacionadas con el sector primario: agrario-forestal, ganadería, pesca y minería.

Estudios universitarios: Ingeniería Superior: Agrónomo; Montes; Minas. Ingeniería Técnica: Hortofruticultura y Jardinería; Industrias Forestales. Ciencias del Mar; Ciencias Ambientales.

Ciclos Formativos Familias Profesionales de: Agricultura y ganadería; Actividades marítimo Pesqueras e Industrias alimentarias.

Profesiones como: Agricultor; Ganadero; Maderero; Pescador; Piscicultor; Vinicultor.

\section{ITEM 19 19. DEPORTE:}

Interés por profesiones relacionadas con la práctica y/o la enseñanza de actividades gimnásticas y deportivas en todos los periodos de edad.

Estudios universitarios: Ciencias de la Actividad Física y Deporte; Magisterio en Educación Física.

Ciclos Formativos Familia Profesional de: Actividades Físico-deportivas. Profesiones como: Deportista; Entrenador; Arbitro; Preparador físico; Profesor educación física; Monitor deportivo.

Revisa tus respuestas en el cuestionario y escribe en el recuadro de entre las diecinueve áreas cual es en este momento tu AREA VOCACIONAL DE INTERES PREFERENTE, esto es, aquella donde tu encuentres la profesión o estudios que te gustaría realizar en un futuro próximo:

20. En relación con las posibilidades reales que tu crees tener para poder realizar finalmente alguno de los estudios o actividades profesionales de tu interés perteneciente a esta área vocacional:

A. Pienso que nunca llegare a desempeñarla.

B. Creo que tengo muy pocas posibilidades

C. Pienso que es probable que pueda realizarla

D. Tengo una gran seguridad en conseguirlo

21. De acuerdo con mis respuestas en el Cuestionario de Áreas de Intereses Básicos Académico Profesionales realizado anteriormente, mi área vocacional de interés preferente se encuentra: 

A. Entre las que me son indiferentes o tengo dudas.
B. Entre las que me gustan bastante.
C. Entre mis tres o más preferidas.
D. Entre mis dos preferidas.
E. Es mi única preferida.

Fecha de recepción: 28-02-03

Fecha de revisión: 26-02-04

Fecha de aceptación: 12-05-04 